\title{
Impacto de la promoción de la salud en el lugar de trabajo
}

\section{The effectiveness of workplace health promotion}

\author{
Consol Serra ${ }^{1,2,3}$ \\ Elena Ronda ${ }^{3,4}$ \\ ${ }^{1}$ CiSAL-Centro de Investigación en Salud Laboral, IMIM/UPF, Barcelona, España. \\ ${ }^{2}$ Servicio de Salud Laboral, Parc de Salut Mar, Barcelona, España. \\ ${ }^{3}$ CIBERESP, Madrid, España \\ ${ }^{4}$ Grupo de Investigación en Salud Pública, Universidad de Alicante, Alicante, España.
}

\section{Resumen}

Este trabajo es un comentario del artículo: Proper KI, van Oostrom SH. The effectiveness of workplace health promotion interventions on physical and mental health outcomes - a systematic review of reviews. Scand J Work Environ Health. 2019;45(6):546-559. doi: 10.5271/ sjweh.3833.

\section{Abstract}

This text is a commentary on the article: Proper $\mathrm{KI}$, van Oostrom SH. The effectiveness of workplace health promotion interventions on physical and mental health outcomes - a systematic review of reviews. Scand J Work Environ Health. 2019;45(6):546-559. doi: 10.5271/ sjweh.3833.

Fechas · Dates

Recibido: 10/01/2022

Aceptado: 14/01/2022

Publicado: 17/01/2022

\section{Sección coordinada por · Section coordinators}

Consol Serra (consol.serra@upf.edu) Ma del Mar Seguí (mm.Segui@ua.es) 


\section{Resumen del artículo comentado}

Objetivo: Esta revisión sistemática tuvo como objetivo proporcionar una visión general de la efectividad de las intervenciones de promoción de la salud. en el lugar de trabajo sobre los resultados de salud física y mental relacionados con las enfermedades crónicas

Métodos: Se realizó una búsqueda de revisiones publicadas entre 2009 y 2018 en bases de datos electrónicas. Referencias de las revisiones incluidas se revisaron en busca de revisiones adicionales. Intervenciones de promoción de la salud en el lugar de trabajo fueron incluidos si estudiaron los factores de riesgo metabólicos como importantes predictores de diabetes mellitus tipo 2 (T2DM) y enfermedades cardiovasculares (ECV) o si estudiaron resultados de salud mental o musculoesquelética. Revisar la calidad se evaluó utilizando la lista de verificación AMSTAR.

Resultados: De las 23 revisiones incluidas, 9 fueron de alta calidad. Para los resultados relacionados con el peso, hubo una fuerte evidencia de los efectos favorables de las intervenciones en el lugar de trabajo, especialmente para las intervenciones dirigidas a la actividad física y/o dieta. Para los factores de riesgo metabólicos restantes, no hubo evidencia de un efecto positivo de las intervenciones de promoción de la salud debido a la ausencia de revisiones de alta calidad y conclusiones mixtas entre revisiones. También hubo pruebas sólidas de un pequeño efecto positivo en la prevención de los trastornos de salud mental con intervenciones psicológicas en el lugar de trabajo, especialmente aquellas que utilizan técnicas de e- salud y la terapia cognitiva conductual. Además, se encontró una fuerte evidencia para la prevención de los trastornos musculoesqueléticos a través de intervenciones en el lugar de trabajo, especialmente el entrenamiento con ejercicios de resistencia.

Conclusiones: Esta revisión encontró evidencia de la efectividad de las intervenciones en el lugar de trabajo para la prevención de efectos relacionados con el peso, así como la salud mental y los trastornos musculoesqueléticos. Sin embargo, es necesaria investigaciones futuras sobre los factores que contribuyen al éxito de la implementación de una intervención.

\section{Comentario}

En las últimas décadas, y principalmente por la mejora de las condiciones de trabajo, la evidencia científica es muy clara sobre los efectos beneficiosos del trabajo sobre la salud, siempre que las condiciones en las que este se realice sean razonablemente buenas ${ }^{(1,2)}$. Esto es así porqué el trabajo, además de ingresos económicos que contribuyen a proporcionar las necesidades básicas y unas mejores condiciones de vida, aporta una identidad social, estímulos y satisfacciones, relaciones sociales, proyecta la creatividad, contribuye de forma importante a la organización del tiempo, permite el desarrollo de las propias capacidades, conocimientos y expectativas, entre otros. Por otro lado, gozar de un buen estado de salud permite a las personas mantenerse en el trabajo y eso a su vez repercute 
positivamente sobre su salud. Es lo que podemos llamar un círculo virtuoso cuya consecuencia, entre otros, es contribuir a lo que ya es una realidad contrastada científicamente de que cuántos más años trabajes, más años vivirás (the longer you work, the longer you live)(3). Eso sí, como decíamos antes y también contrastado científicamente, siempre que las condiciones de trabajo sean razonablemente buenas ${ }^{(2)}$.

Por otro lado, ayudar a las personas a que contribuyan a su propia salud mediante intervenciones de promoción de la salud, especialmente sobre sus propios estilos de vida, forma una parte muy relevante de este proceso. Y como es bien conocido y recomendado por la Organización Mundial de la Salud desde los años '50 el lugar de trabajo ofrece sin duda una gran oportunidad para promover la salud, en este caso, en la población activa laboralmente ${ }^{(4)}$.

Un estilo de vida poco saludable puede contribuir a una mala salud, aumento del absentismo y también a una pérdida de productividad en el trabajo. Los programas de promoción de la salud en el lugar de trabajo tienen como objetivo mejorar el estilo de vida y, en consecuencia, mejorar la salud. Sin embargo, los estudios de intervención muestran resultados pocos concluyentes, y la evaluación general de la efectividad está obstaculizada por una gran heterogeneidad en las intervenciones y las poblaciones de estudio(3).

Para que las intervenciones de promoción de la salud sean realmente efectivas en salud deben reunir una serie de condiciones. En las últimas décadas es mucha la evidencia científica publicada sobre diferentes estrategias de intervenciones de promoción de la salud en el lugar de trabajo, lo que ha permitido realizar revisiones sistemáticas. La investigación que se presenta aquí consiste en una revisión de revisiones sistemáticas (una overview) que aborda no sólo estudios individuales de calidad sino que da un paso adelante al seleccionar revisiones sistemáticas, que ya han seleccionado la evidencia disponible de calidad, y que al ser evaluadas mediante herramientas sistematizadas y contrastadas, como es la escala AMTSAR, aporta un material excelente para proporcionar conclusiones y recomendaciones para la implementación de intervenciones en este caso en el lugar de trabajo con el objetivo de promocionar la salud de las personas trabajadoras ${ }^{(5)}$.

Es interesante que los autores se centran sobre todo en el efecto de la promoción de la salud en la salud mental y los trastornos musculoesqueléticos, bien conocidos como las dos causas que mayor morbilidad y discapacidad ocasionan en la población laboral. El equipo de revisión identificó 374 citas a partir de las cuales se identificaron 23 revisiones sistemáticas que cumplian los criterios de inclusión. La evaluación de la calidad científica de estas revisiones fue evaluada de forma sistemática, y tan solo 9 fueron de alta calidad, el resto se consideraron de baja calidad. Los resultados analizados estaban relacionados con el peso corporal, el riesgo metabólico, la salud mental y la salud osteomuscular, de manera que solo para estos últimos la mitad o más de las revisiones incluidas en el análisis eran de calidad elevada. 
En cualquier caso, la revisión permite evidenciar los efectos positivos en los resultados relacionados con la pérdida de peso, la mejora de la salud mental y la prevención de los trastornos musculoesqueléticos. De forma específica, la revisión también identifica aquellas intervenciones, entre la gran diversidad, que se muestran como efectivas; por ejemplo, en el caso de la salud mental serían las relacionadas con la terapia conductual, y actividades de e-health. No obstante, como destacan los autores muchas de las intervenciones son multimodales, e identificar cuáles son realmente efectivas es difícil con los diseños utilizados. Para el resto de las intervenciones analizadas sobre los factores de riesgo cardiometabólico, excepto medidas relacionadas con el peso, la evidencia fue inconsistente, en parte atribuible a que muchos estudios tienen periodos de seguimiento cortos para evaluar enfermedad cardiovascular o diabetes.

Sería necesario desarrollar investigaciones en los que se evalúen las barreras y facilitadores para el acceso de los trabajadores a este tipo de intervenciones y sirvieran para dar información de utilidad tanto a las empresas como a los servicios de prevención para ponerlas en práctica.

\section{Bibliografía}

1. Butterworth P, Leach LS, Strazdins L, et al. The psychosocial quality of work determines whether employment has benefits for mental health: results from a longitudinal national household panel survey. Occup Environ Med. 2011;68(11):806-12. doi: 10.1136/oem.2010.059030.

2. van der Noordt $M$, IJzelenberg $H$, Droomers $M$, et al. Health effects of employment: a systematic review of prospective studies. Occup Environ Med. 2014;71(10):730-6. doi: 10.1136/oemed-2013-101891.

3. Rongen A, Robroek SJW, van Lenthe FJ, et al. A. Workplace health promotion: a meta-analysis of effectiveness. Am J Prev Med. 2013;44(4):406-415. doi: 10.1016/j.amepre.2012.12.007.

4. Burton, J. WHO Healthy workplace framework and model: Background and supporting literature and practices. World Health Organization. [actualizado 14 ene 2022; citado 14 ene 2022]. Disponible en: https://apps.who.int/iris/handle/10665/113144

5. Proper $\mathrm{KI}$, van Oostrom SH. The effectiveness of workplace health promotion interventions on physical and mental health outcomes - a systematic review of reviews. Scand J Work Environ Health. 2019;45(6):546-559. doi: 10.5271/sjweh.3833. 